\title{
Social Interaction Test in Home Cage as a Novel and Ethological Measure of Social Behavior in Mice
}

\author{
Do Gyeong Kim ${ }^{\dagger}$, Edson Luck Gonzales ${ }^{\dagger}$, Seonmin Kim, \\ Yujeong Kim, Keremkleroo Jym Adil, Se Jin Jeon, \\ Kyu Suk Cho, Kyoung Ja Kwon* and Chan Young Shin* \\ Department of Neuroscience, School of Medicine and Center for Neuroscience Research, \\ Konkuk University, Seoul 05029, Korea
}

\begin{abstract}
Sociability is the disposition to interact with one another. Rodents have a rich repertoire of social behaviors and demonstrate strong sociability. Various methods have been established to measure the sociability of rodents in simple and direct ways, which includes reciprocal social interaction, juvenile social play, and three-chamber social tests. There are possible confounding factors while performing some of these tasks, such as aggression, avoidance of interaction by the stimulus mouse, exposure to a new environment, and lengthy procedures. The present study devised a method to complement these shortcomings and measure sociability as a group in the home cage setting, which prevents group-housed mice from isolation or exposure to a new environment. The home cage social test can allow high-throughput screening of social behaviors in a short amount of time. We developed two types of home cage setup: a home cage social target interaction test that measures sociability by putting the wire cage in the center area of the cage and a home cage two-choice sociability and social preference test that measures both sociability or social preference by putting cage racks at opposite sides of the cage. Interestingly, our results showed that the two types of home cage setup that we used in this study can extract abnormal social behaviors in various animal models, similar to the three-chamber assay. Thus, this study establishes a new and effective method to measure sociability or social preference that could be a complementary assay to evaluate the social behavior of mice in various setup conditions.
\end{abstract}

Key words: Home cage, Sociability, Novel environment, Social test

\section{INTRODUCTION}

Received October 31,2018, Revised March 18,2019,

Accepted April 2, 2019

* To whom correspondence should be addressed.

Chan Young Shin, TEL: 82-2-2030-7834, FAX: 82-2-2049-6192

e-mail: chanyshin@kku.ac.kr

Kyoung Ja Kwon, TEL: 82-2-454-5630, FAX: 82-2-2049-6192

e-mail: neuro11@kku.ac.kr

These authors contributed equally to this work.
Social behavior is the natural disposition to interact or associate with one another [1]. Members of a society share information with peers, express emotions, empathize, and maintain a connection with each other through various means of communication. Social behavior itself is complex, yet important, to the health and wellbeing of every individual $[2,3]$. Thus, the understanding of social behavior and the mechanisms of its impairment in various disor- 
ders is vital.

Deficits in social behavior can result in lifelong problems, and the disorder most associated with social deficits is autism spectrum disorder (ASD) [4-10]. Social deficits are mainly described as having less interest in peers, difficulty keeping up with social interactions, and difficulty using verbal and nonverbal communication [11-13]. The social deficits found in individuals with autism remain difficult to manage, primarily because of limited understanding of the complex mechanisms of social behavior in general. Obviously, the unresolved issue of how to improve social deficits in ASD calls for deeper understanding and research on social behavior as a whole.

The social interaction aspect of social behavior is a common measurement to assess the social impairment criterion relevant to ASD [14]. While the social interaction behavior of humans is generally more broad and complex than that of animals, the use of screening tools to at least partially reflect this behavior in animal models have contributed to the understanding of the underlying mechanisms of social behavior in the brain [15-19]. The reciprocal social interaction test and juvenile social play are common measures of direct social interaction in mice [20]. These tests are able to extract the naturalistic social behavior phenotypes of subject mice to a stimulus mouse by direct contact. The types of social behaviors commonly observed have been identified as nose-to-nose or oral-to-oral contact, nose-to-genital or anal sniffing, following, pushing over, and crawling under [21-23]. The usual set-up of this experiment involves habituating the subject to a new cage-sized arena, with bedding to establish a territory, before introducing the stimulus mouse. In some cases, subjects that have been singly housed could directly be tested with a stimulus mouse in its home cage. The analysis would usually require manual observation of these behaviors and require some level of experience and clarity to measure and quantify the different types of social interaction. A possible confound of direct social interaction is that the stimulus mouse can initiate or avoid interactions with the subject mouse, as it can move freely in the arena [20]. Another variable is the possible occurrence of aggressive behaviors by the subject mouse to the stimulus mouse or vice versa. Thus, the three-chamber social test was developed to potentially address those issues.

The three-chamber sociability and social preference for novelty assays developed by Crawley's team [24,25] are frequently used tools to assess the social interaction of subject mice to an enclosed conspecific mouse inside a small wire cage. The main parameter of this test was time spent by the subject mouse in the chamber containing the conspecific stranger in a wire cage, versus the chamber containing an empty wire cage. This test has the advantage of letting the subject initiate the social approach to the enclosed stimulus and prevent aggressive contact. The social preference for novelty test assesses the tendency of the test mouse to explore a more novel mouse over an already familiar one. This has implications for social recognition and aspects of memory, and might be implicated in ASD [26]. While this test is merited as useful and highly controlled, there are aspects of this test which would benefit from a complementary social behavior paradigm. One problematic aspect is the exposure of subject mice to a new environment during the test, which contributes a confounding effect of noveltyinduced behavioral change to the social behavior being measured [27]. Other aspects to improve include the simplicity and time efficiency in performing the test, since the three-chamber social task requires a long trial duration and experimental period for the entire set of mice tested.

The use of a home cage condition is a meaningful tool to assess mouse behaviors in a less stressful and more naturalistic setting [28, 29]. Some researchers have attempted to measure the home cage social behavior of mice between paired cage mates, and compared their sociability in a social interaction test [27]. They found that the paired cage mates in the home cage were more involved in passive social behaviors than active interactions. On the other hand, testing those mice in the three-chamber sociability paradigm, where a novel stimulus mouse was introduced, initiated more active social exploration. This was also true in reciprocal social interaction tasks, social novelty preference tests, and social memory tasks; active social interactions of the subject mouse to a novel stimulus mouse were noted, whilst there was less interaction with a familiar mouse $[20,25,30]$.

In order to unify both social test conditions, the current study aimed to develop a novel social interaction paradigm in a familiar and less stressful environment by testing the group-housed mice in their home cage. The stimulus mouse will be introduced through a wire cage to let the subject mice initiate the social interaction. Our study sought to establish a group-housed type of social interaction test in the home cage of mice to save space and time in terms of maintenance and conducting the experiment itself. These modifications are in contrast to a previous study which first described a modified resident-intruder assay in single-housed animals [31]. Through the use of several mouse models of social deficit, we are able to determine the efficiency of the home cage social interaction test in line with an already established assay. According to the results, this test can promote high throughput testing and can be used as a new screening tool to assess social interaction in a home cage setting simultaneously for group-housed mice. 


\section{MATERIALS AND METHODS}

\section{Animals}

Three types of animal models were used in this study, and only male mice were used for the experiments. First, the Cntnap2 gene knockout mice (JAX 017482 B6.129(Cg)-Cntnap2tm1Pele/J) were acquired from The Jackson Laboratory (Maine, USA). These mice were bred in the animal facility. The following day after mating, the vaginal plug was checked to designate as E0. Female mice were housed with their litters until the weaning period (P21). To identify the genotype of each animal using PCR, genomic DNA was extracted from the tail at P14. The other two models were drug-induced (NMDA and MK-801 injection) juvenile ICR male mice purchased from Orient Bio Inc. (Korea) at 3 weeks of age and habituated to the lab for at least 5 days before the commencement of behavioral experiments.

All procedures, including animal handling, were in accordance with the approved Animal Care and Use Guidelines of Konkuk University, Korea (KU18054) and were in compliance with the National Institute of Health Guide for the Care of Laboratory Animals (NIH Publications No. 8023, revised 1978). All subjects were housed on a 12:12-h circadian cycle (lights on at 00:00 and off at $12: 00)$, at a constant temperature $\left(22 \pm 2^{\circ} \mathrm{C}\right)$ and humidity $(55 \pm 5 \%)$, with food and water ad libitum.

\section{Treatments}

The N-methyl-D-aspartate (NMDA, Product No. M3262-1g) and (+)-MK-801 hydrogen maleate (MK-801, Product No. M107$50 \mathrm{mg}$ ) were obtained from Sigma-Aldrich and were dissolved in normal saline $(0.9 \% \mathrm{v} / \mathrm{v})$ to be injected via intraperitoneal routes. The volume of injection for each injected drug was $10 \mathrm{ml} / \mathrm{kg}$, while the concentration varied depending on dosage: NMDA solution at $5 \mathrm{mg} / \mathrm{ml}$ and MK-801 solution at $0.015 \mathrm{mg} / \mathrm{ml}$. NMDA and MK-801 were injected directly into wild-type juvenile mice at 50 and $0.15 \mathrm{mg} / \mathrm{kg}$, respectively, $30 \mathrm{~min}$ before each test. The control groups were given saline as the vehicle.

\section{Behavioral observation}

All behavioral experiments were performed during the dark phase from 13:00 to 18:00. Experiments were performed from 3 to 5 weeks of age in ICR mice and from 6 to 7 weeks of age in C57BL/6 mice. Subject mice were habituated in the test room at least $30 \mathrm{~min}$ before each actual experiment began. Behavioral experiments were performed under a dim light condition (3 lux, incandescent lamp). In the social tests, the stimulus mice were of the same strain, with no previous encounter with the subject mice, and their weights were lower by an average of $2 \mathrm{~g}$.

\section{Three chamber sociability and social novelty preference tests}

The test was performed in three connected rectangular chambers $(230 \times 400 \times 220 \mathrm{~mm}$ in each compartment). Two openings measuring $9 \mathrm{~cm}^{2}$ provided access to each compartment. The central compartment was the starting point for each test. Wire cages with $10 \mathrm{~cm}$ diameter were placed in the middle of each side compartment. The subject mouse was allowed to roam around the three chambers for 5 minutes as a habituation period. Then, the sociability test commenced by putting the stranger (stimulus) mouse into one of the two wire cages. and the other wire cage was left empty. The subject was then allowed to explore the three chambers for 10 minutes. The experiment operationalized sociability by measuring the time spent by the subject mouse between the empty and stranger wire cage compartments. Immediately after, the social novelty preference test commenced for an additional 10 minutes, where a new stranger (stimulus) mouse was added to the previously empty wire cage, while the previous stranger mouse became the familiar mouse. After the entire trial, the chambers and wire cages were cleaned with $70 \%$ ethanol. EthoVision software (EthoVision 3.1, Noldus Information Technology, Netherlands) was linked via an overhead camera and tracked the movement of each subject in every compartment. The duration of stay in each compartment and the duration of stay in the approach area $(5 \mathrm{~cm}$ around the wire cage) was measured automatically by EthoVision.

\section{Home cage social target interaction test}

The subject mice were housed in groups of five in a large home cage $(260 \times 420 \times 180 \mathrm{~mm})$ at least 3 days before the test commenced. In most cases, those mice had already been previously housed together in smaller cages but were transferred to a larger one for the test accommodation. Two cages were tested simultaneously per trial, with an opaque cover in between to prevent visual cues from other mice. The wire cage used for the stranger mouse was the same size and dimensions used for the three-chamber social test. An empty wire cage was placed in the middle of each home cage for a 30 -minute familiarization period. After this, a stranger mouse was placed in the wire cage, and the behavior of test mice was recorded by an overhead camera for 15 minutes (Fig. 1A). We ensured that the overhead camera resolution was clear enough $(640 \times 480)$ to capture the sniffing of mice to the wire cage, and we zoomed as close as possible while maintaining a view of the entire cage area. Please refer to the supplementary movie file for the video samples. The sniffing duration to the wire cage was scored manually by a blind observer during the 15 -minute trial period. Sniffing was noted when the subject mouse's nose was in close contact and obviously interacting with the wire cage, where 
A.

Stimulus (stranger) mouse
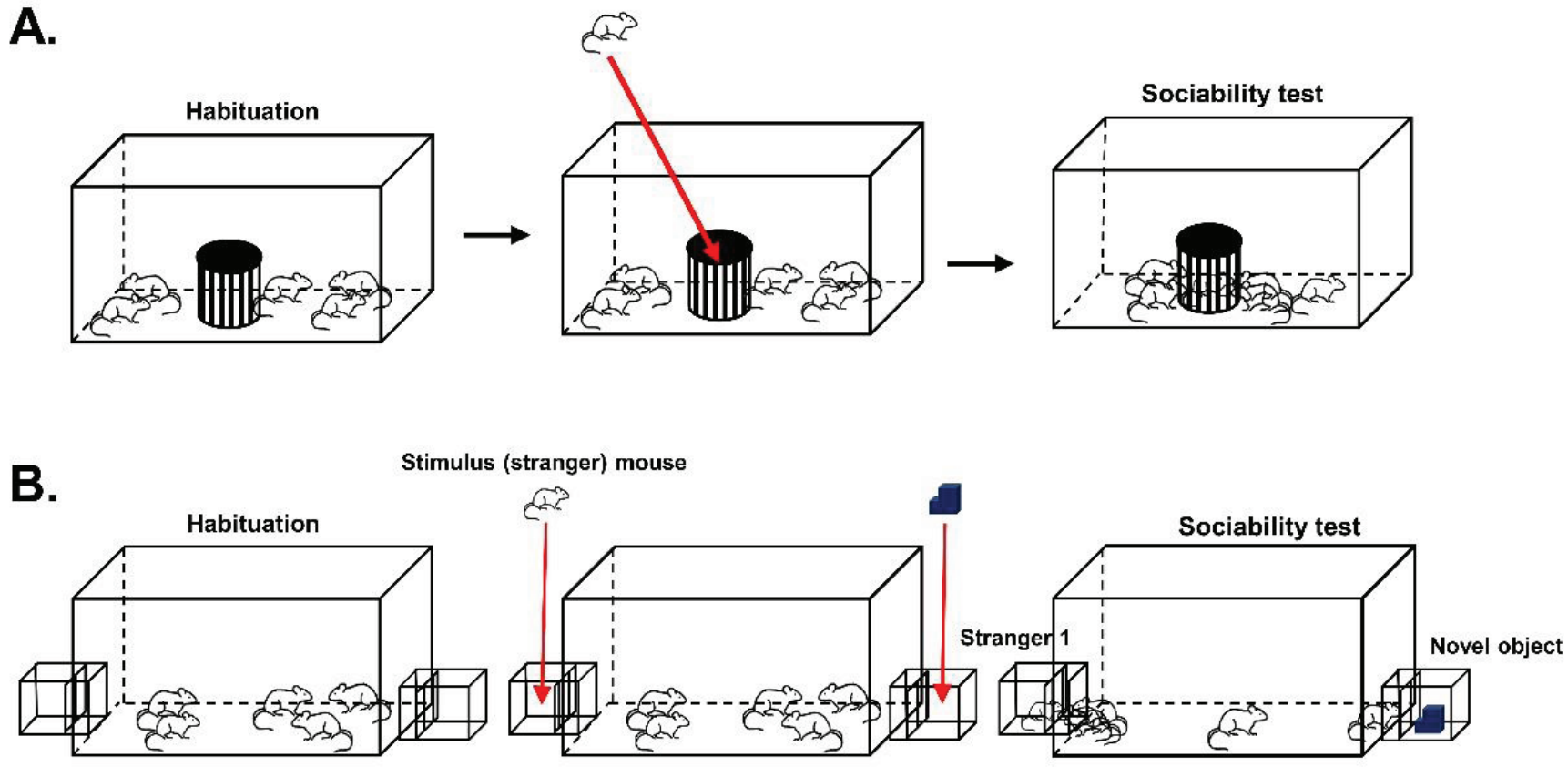

C.
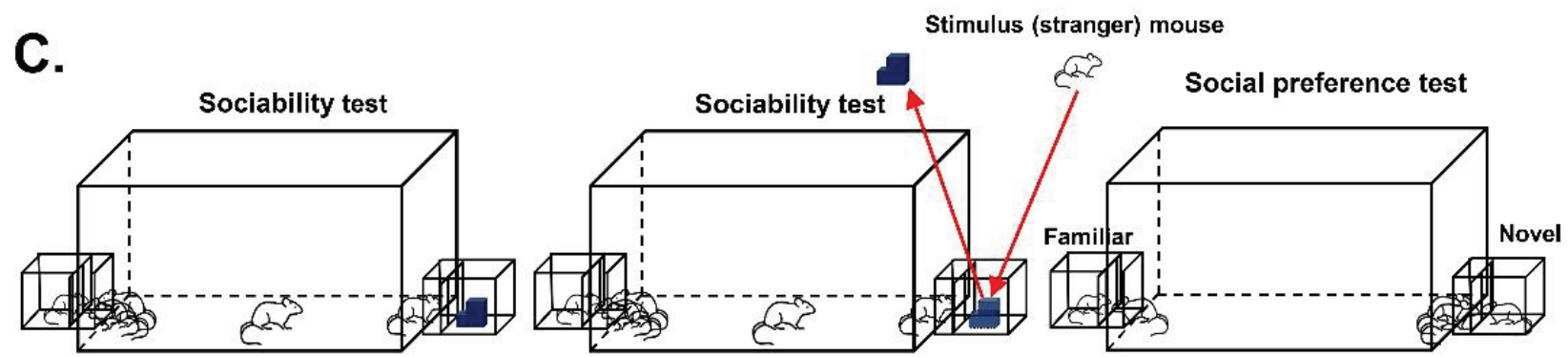

D.

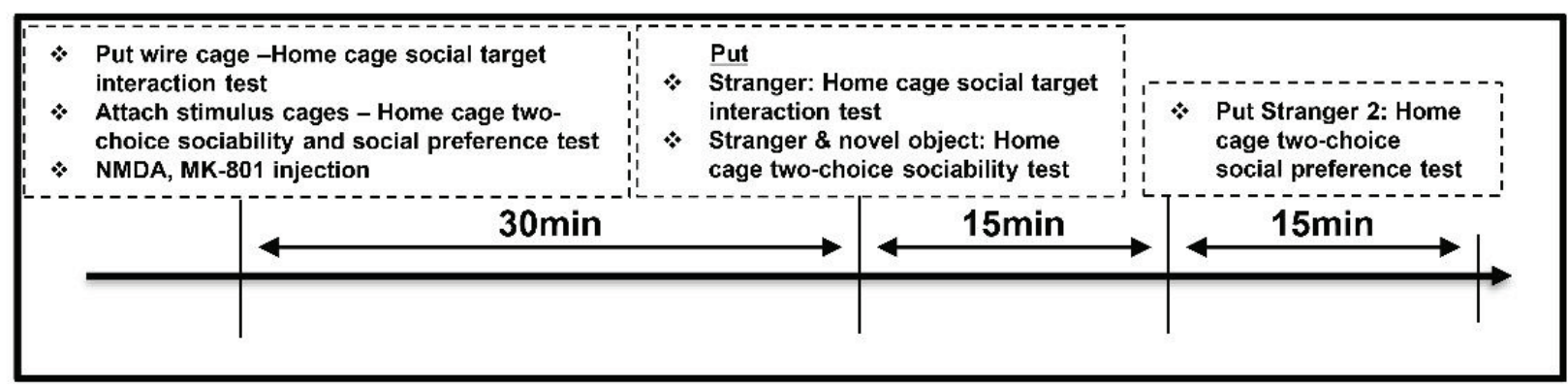

Fig. 1. Social interaction test in home cage, social target interaction test, and home cage two-choice sociability test methods - (A and B) sociability tests;(C) transition to social novelty preference test; (D) the experiment scheme.

the stimulus mouse was located. Leaning of subject mice toward the wire cage while the nose was pointing somewhere else was not counted as a sniffing behavior. The individual mice in the home cage social interaction tests were tracked based on their tail marks using a dot or line numbering system, where one dot equals to one and so on, and a line represents the number 5 . We used a black ink permanent marker (namepen) to mark the tail visibly enough to be seen in the video recordings. 
Home cage two-choice sociability and social preference test

In this experiment, the subject mice were housed in groups of five in a modified large home cage $(260 \times 420 \times 180 \mathrm{~mm})$. Habituation and testing conditions were similar to those of the home cage social target interaction test. The home cage two-choice sociability and social preference test home cage had a rectangular hole in both distant walls, where the stimulus mouse cages can be attached during the test (Fig. 1B and 1C). During the housing and maintenance period, the wall openings were covered by a clear plexiglass similar to the home cage material. The stimulus cage $(140 \times 140 \times 100 \mathrm{~mm})$ used to house the stranger mouse during the test was made of a transparent polyvinyl chloride that could be tightly attached to the home cage openings. Inside the stimulus cage was a wire mesh located $20 \mathrm{~mm}$ from the connection point of the home cage to prevent full body contact between subject and stimulus mice. Before the actual trials, the two empty stimulus cages were attached to the sides of the home cage, and the opening covers removed as it was placed in the experimental area for 30 minutes habituation. Then, a stranger mouse was placed in one of the stimulus cages, while an inanimate object on the other side was used to measure the social interaction in a home cage setting for $15 \mathrm{~min}$ (Fig. 1B). This test was also followed by the social novelty preference test for an additional 15 minutes as a new stimulus (novel) mouse replaced the inanimate object (Fig. 1C). The whole trial was recorded by an overhead camera for 15 minutes, similar to that of the home cage social target interaction test. In the analysis, the sniffing duration to stimulus cages was measured manually by blind observers during the 15-minute trial period, and each mouse was individually identified through tail markings.

\section{Statistical analysis}

All data were presented as the mean \pm SEM. Statistical analyses were conducted using GraphPad Prism software version 5.03 for Windows. Statistical analyses included a one-way analysis of variance (ANOVA) followed by Tukey post hoc test analysis. Where two variables were compared, two-way ANOVA was used fol-
A.

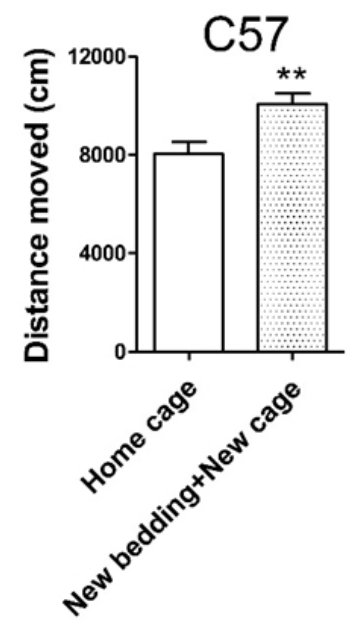

C.

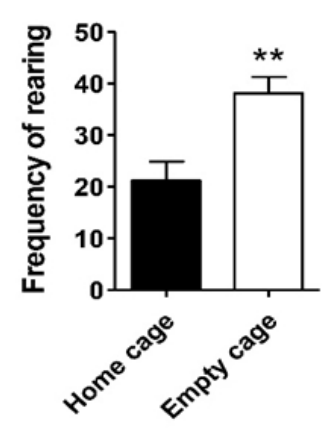

B.

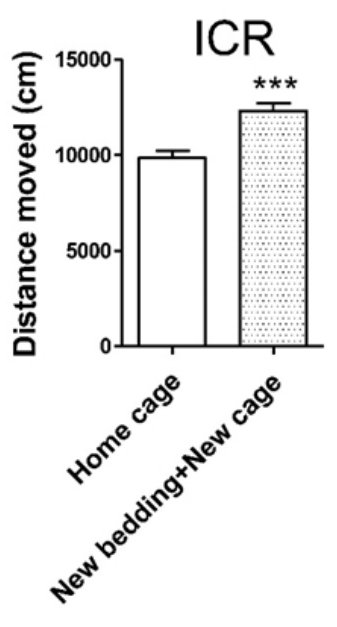

D.

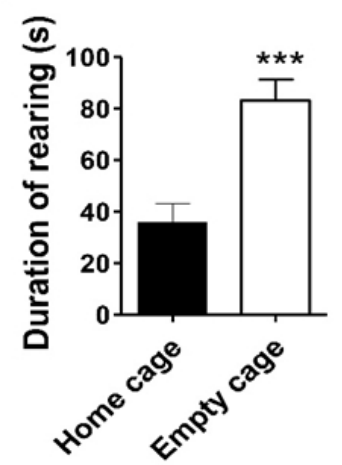

E.

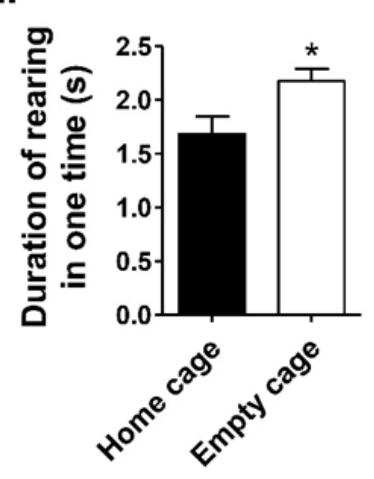

Fig. 2. Effect on locomotor activity and exploratory behavior of home cage and new cage environment-exposed mice. The distance moved of (A) C57BL/6 mice and (B) ICR mice in the home cage or new bedding+new cage (home cage, $n=9$, and $\mathrm{N}+$ $\mathrm{N}=$ new bedding + new cage, $\mathrm{n}=9$ ). The frequency $(\mathrm{C})$, accumulated duration (D) and average duration per episode $(\mathrm{E})$ of rearing or leaning toward the cage walls of the home cage and empty cage housed mice during the social interaction test (home cage, $n=9$ and empty cage, $n=10$ ). All data are expressed as the mean \pm SEM. ${ }^{*} \mathrm{p}<0.05,{ }^{* *} \mathrm{p}<0.01,{ }^{* * *} \mathrm{p}<0.001$ vs. home cage housed mice. 
lowed by Bonferroni post hoc comparisons. Unpaired t-tests were also used to calculate the difference between control and animal model groups. If the $\mathrm{p}$-value was less than 0.05 ( $\mathrm{p}<0.05)$, the difference was considered statistically significant.

\section{RESULTS}

\section{Novel environment exposure affects the locomotor and ex- ploratory activity of control mice}

To check the effect of novel environment exposure on subject mice, locomotor activity was evaluated. Fifty percent of the animals raised in the same cage were randomly selected and remained in the home cage, whilst the remainder were moved to a new cage with new bedding, and their locomotor activity measured for 25 $\min$.

In C57BL/6 mice, the novel environment conditions (new cage with new bedding) resulted in the mice moving greater distances than in the home cage setting (Fig. 2A, p=0.0063). The outbred ICR mice in the new cage exposed group also moved a significantly greater distance than those in the home cage tested group (Fig. 2B, $\mathrm{p}=0.0004$ ). This result indicates that exposure to a new environment affects the locomotor activity of mice.

Exploratory behavior (Fig. 2C, 2D and 2E), marked by rearing and leaning toward the cage walls (not including leaning and rearing toward the stimulus wire cage), significantly increased; the frequency and duration of rearing were noted in new empty cageexposed mice compared with the home cage mice. During the 15min trial, the frequency (Fig. 2C, $\mathrm{p}=0.0028$ ), accumulated duration (Fig. 2D, $\mathrm{p}=0.0004$ ), and average duration per incidence (Fig. 2E, $\mathrm{p}=0.0232$ ) of rearing significantly increased. This result suggests that the new environment can also affect the exploratory behaviors of mice.

\section{Interaction with a stimulus mouse versus a novel object in the home cage condition}

To operationalize the social interaction of subject mice to the stimulus mice in the wire cage by sniffing behavior, we compared the sniffing duration of wild-type mice between two conditions: the presence of a stimulus mouse or a novel inanimate object of a similar size (a Lego ${ }^{\circledR}$ block - plastic $50 \times 30 \times 60 \mathrm{~mm}$ ) in the wire cage. We also measured the sniffing time of the empty wire cage during the last 5 minutes of the 30 minute habituation to establish reduced interest in the wire cage prior to the introduction of the stimulus mouse or object. Indeed, the sniffing time of the wire cage during the last 5 minutes of habituation was lower prior to the increased sniffing during the social test (Fig. 3). In addition, the interaction of subject mice to the stranger in the wire cage is obvi- ously greater than the interaction with a novel object in the wire cage for every time bin during the $15 \mathrm{~min}$ trial period.

\section{NMDA-injected mice exhibit reduced social interaction in both three-chamber and home cage social interaction tests}

This study used an NMDA-injected model as a pharmacological model of social deficits. In the three-chamber test, the NMDAinjected animals spent significantly less time in the stranger compartment compared to their control counterparts (Fig. 4A, $\mathrm{p}=0.0232$ ). The time spent approaching the stranger mouse was also reduced in NMDA-injected mice compared with control mice (Fig. 4B, p=0.0030). Meanwhile, the time spent in the center and the empty wire cage compartment (including approach duration) was not different between the NMDA-injected model and control (Fig. 4A, p>0.05). In the home cage social tests, home cage social target interaction test, and home cage two-choice sociability test, the NMDA-induced model spent less time sniffing the stimulus mouse compared to control counterparts during the entire 15min trial period (Fig. 4C and 4D, p $<0.0001$ in the home cage social target interaction test, and $\mathrm{p}=0.0002$ in the home cage two-choice sociability test). In addition, there was no difference in the duration of sniffing of the object in the home cage two-choice sociability test (Fig. 4D, p>0.05).

The home cage two-choice social preference test was established to accommodate a social novelty preference paradigm. In the three-chamber set up of the social novelty preference test, the NMDA-induced model spent significantly reduced time in the novel mouse area (Fig. 4E, p=0.0390), as well as in the approach

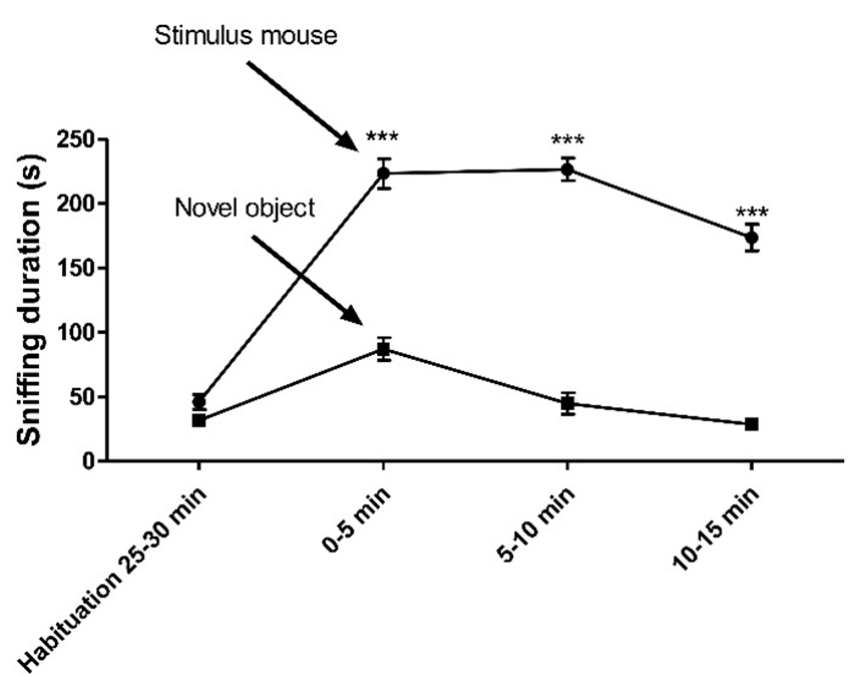

Fig. 3. Sniffing duration by mice of a stimulus mouse versus a novel inanimate object in time bins from the last $5 \mathrm{~min}$ of habituation to $15 \mathrm{~min}$ of introducing the social stimulus. All data are expressed as the mean \pm SEM. ${ }^{* * *}$ is $\mathrm{p}<0.001$ vs. novel object explorations at different time bins $(n=20)$. 
Three chamber

A.

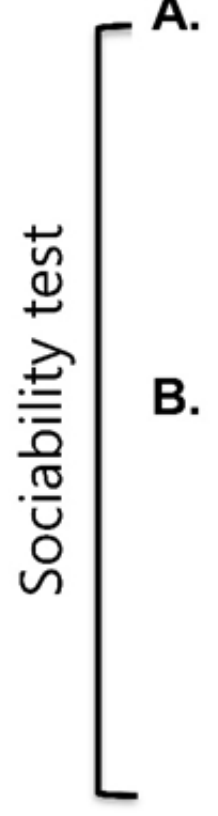

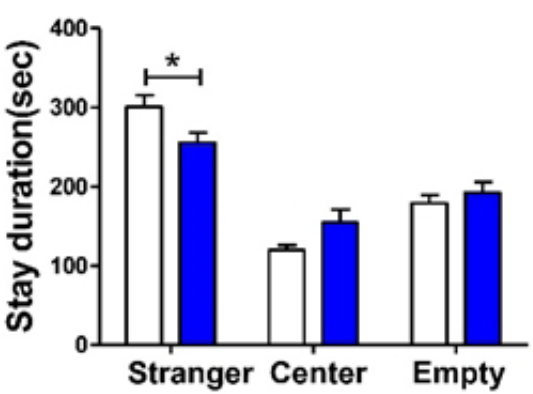

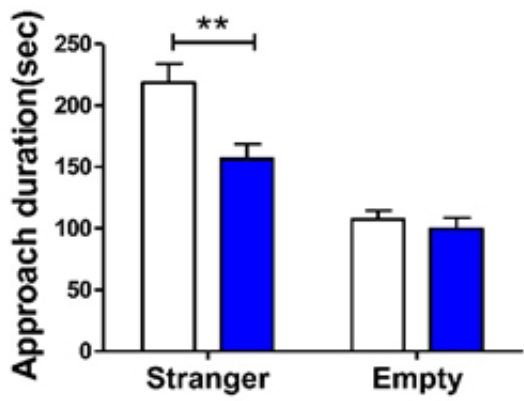

$\mathbf{E}$

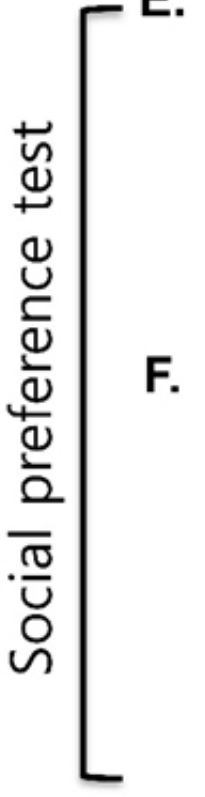

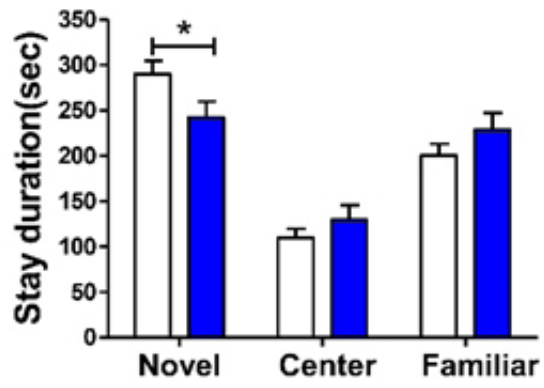

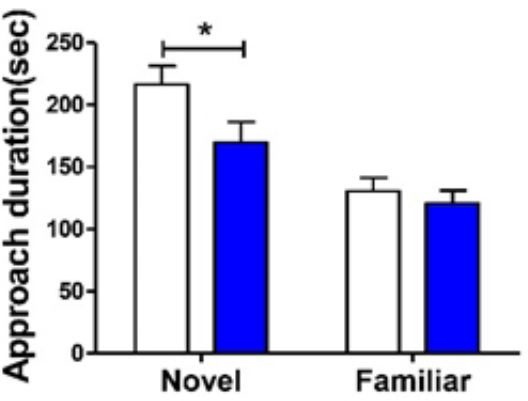

Home cage social test
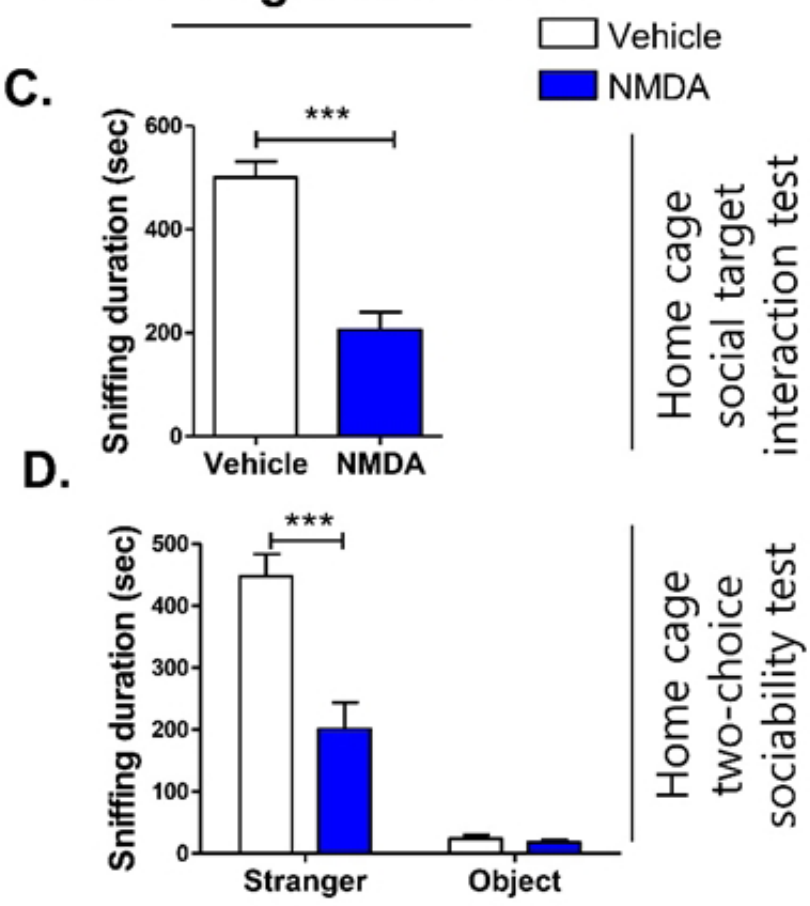

G.

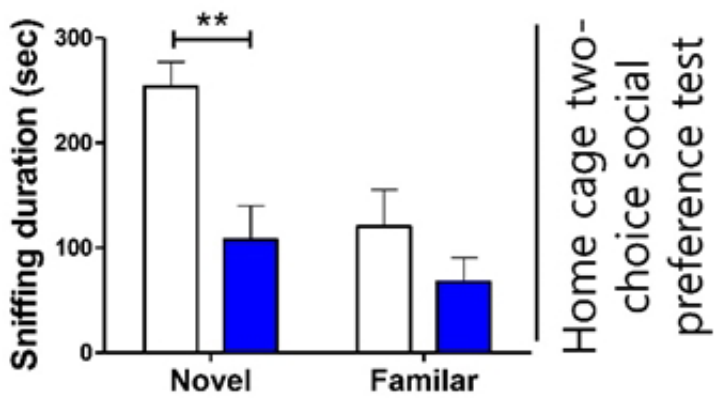

Fig. 4. Sociability and social preference determined by time spent in each compartment and approach duration of NMDA-injected mice model in the three-chamber assay and home cage social test. This figure shows the time spent (duration) in each compartment (A) and approach duration (B) of NMDA-injected mice model compared to controls in the three-chamber social test; the time spent (duration) sniffing by NMDA-injected mice models compared to controls in the home cage social target interaction test (C); the home cage two-choice sociability test (D); the social preference test (E G); the time spent (duration) in each compartment (E) and approach (F) of NMDA-injected mice model compared to controls in the three-chamber social test; sniffing duration in the home cage two-choice social preference test $(\mathrm{G})$. The number of animals per condition was as follows: NMDA and Veh in three-chamber social test, $\mathrm{n}=28$ and $\mathrm{n}=32$, respectively; NMDA and Veh in the home cage social target interaction test, $\mathrm{n}=15$ and $\mathrm{n}=14$, respectively; NMDA and Veh in the home cage two-choice sociability and social preference test, $n=10$. All data are expressed as the mean $\pm S E M .{ }^{*} \mathrm{p}<0.05$, ${ }^{* *} \mathrm{p}<0.01$, ${ }^{* * *} \mathrm{p}<0.001$, Veh, Vehicle group mice; NMDA, NMDA-injected mice. 
A.

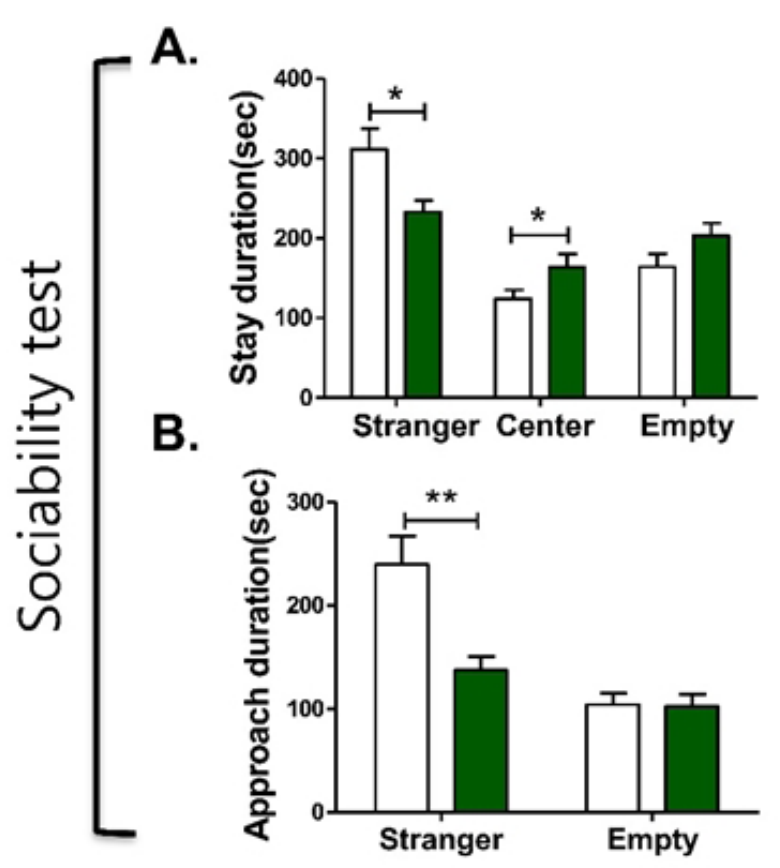

E.

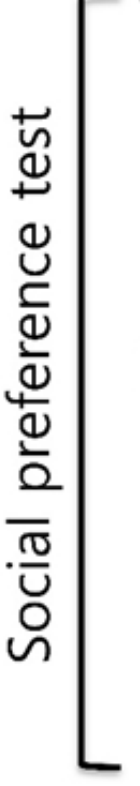

Three chamber
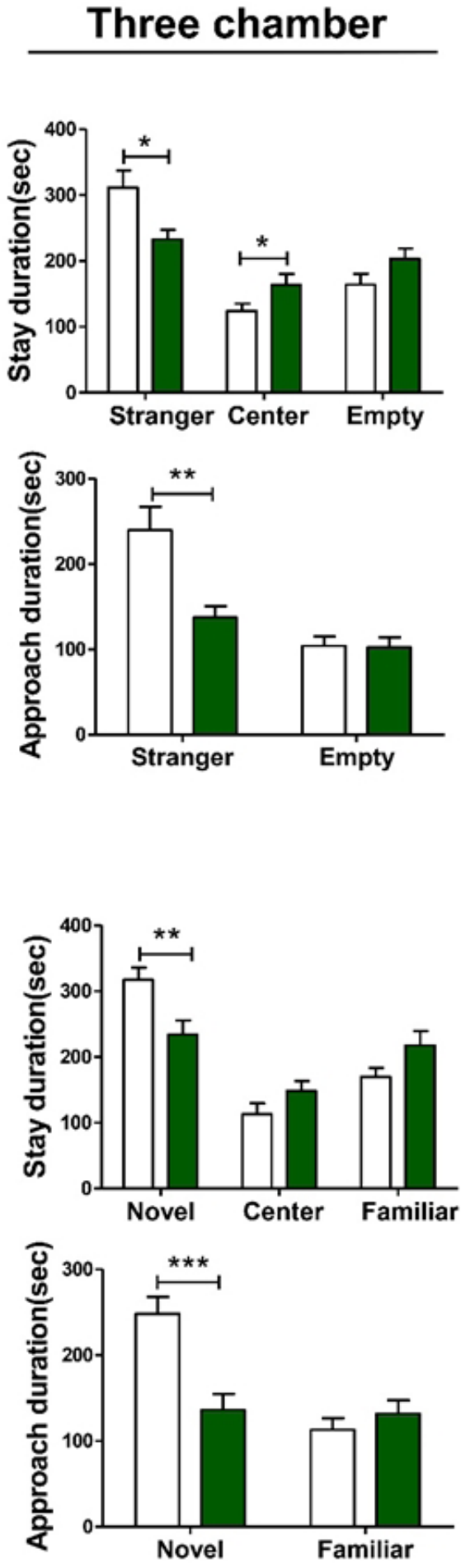

D.

\section{Home cage social test}

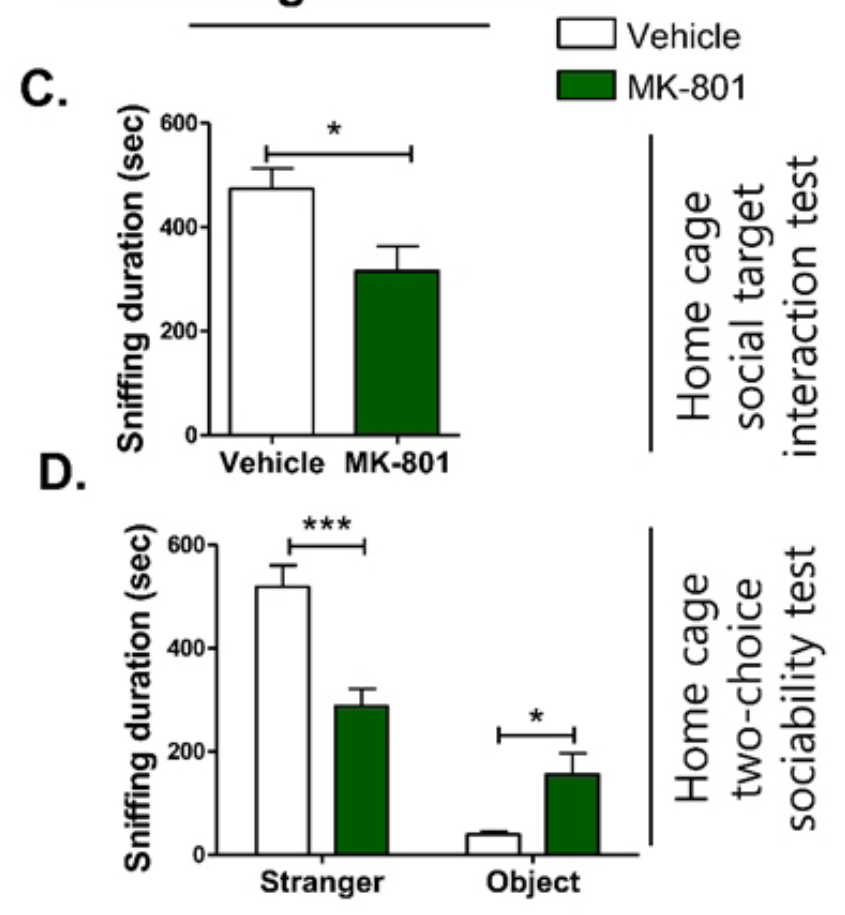

G.

Fig. 5. Sociability and social preference determined by time spent in each compartment and approach duration of MK-801-injected mice model in the three-chamber assay and home cage social test. This figure shows the time spent (duration) in each compartment (A) and approach duration (B) of the MK-801-injected mice model compared to controls in the three-chamber social test; the time spent (sniffing duration) by MK-801-injected mice models compared to controls in the home cage social target interaction test (C); home cage two-choice sociability test (D); the social preference test $(\mathrm{E} \sim \mathrm{G})$; the time spent (duration) in each compartment (E) and approach duration (F) of MK-801-injected mice model compared to controls in the three-chamber social test; the time spent (duration) sniffing by MK-801-injected mice models compared to controls in the home cage two-choice social preference test (G). The number of animals per condition was as follows: MK-801 and Veh in the three-chamber assay, $\mathrm{n}=17$; MK-801 and Veh in home cage social target interaction test, $n=10, M K-801$ and Veh in the home cage two-choice sociability and social preference test, $n=13$ and $n=15$, respectively. All data are expressed as the mean \pm SEM. ${ }^{*} \mathrm{p}<0.05,{ }^{* *} \mathrm{p}<0.01,{ }^{* *} \mathrm{p}<0.001$, Veh, Vehicle group mice; MK-801, MK-801-injected mice. 


\section{Three chamber}

A.

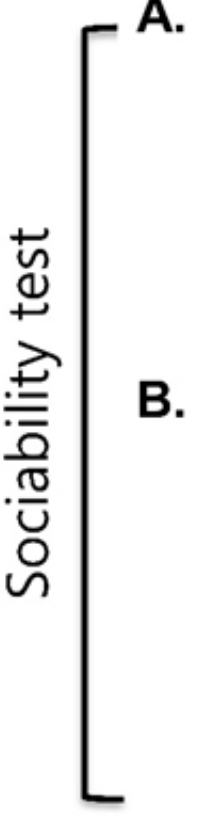

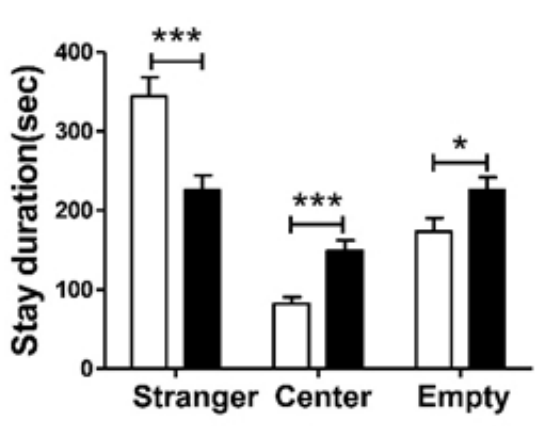

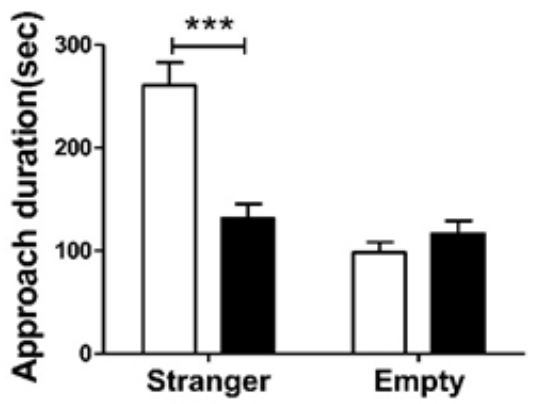

E.

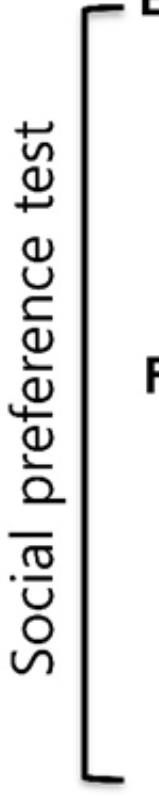

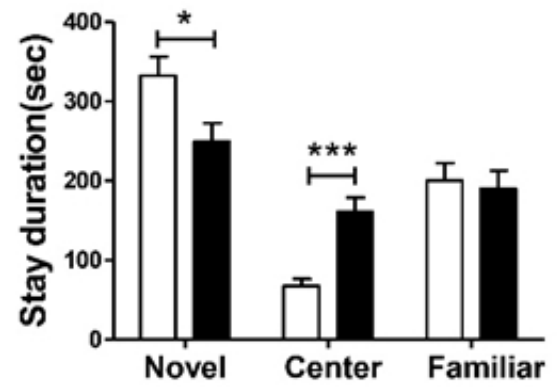

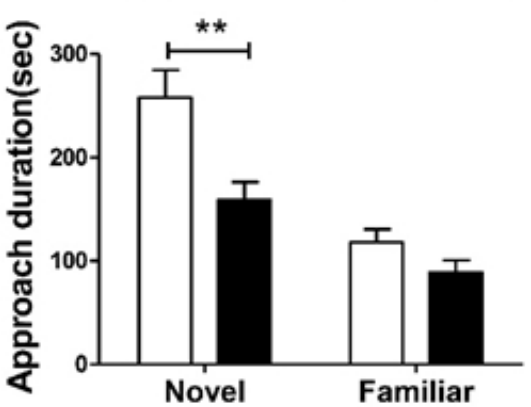

Home cage social test
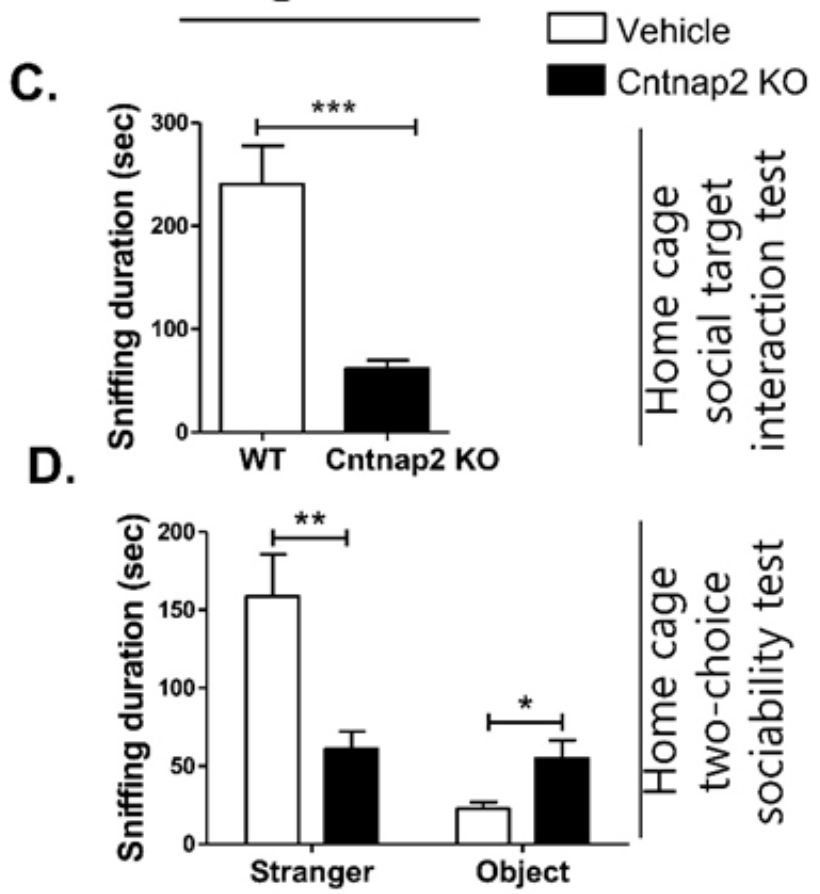

G.

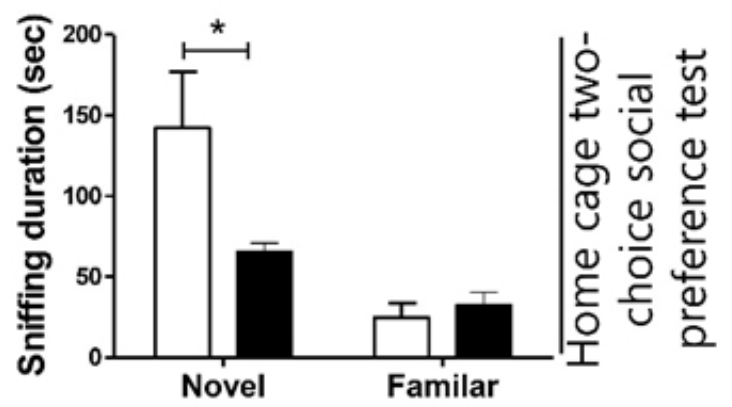

Fig. 6. Sociability and social preference determined by the time spent in each compartment and approach duration of Cntnap2 knockout mice in the three-chamber assay and home cage social test. This figure shows the time spent (duration) in each compartment (A) and approach duration (B) of Cntnap2 knockout mice compared to controls in the three-chamber social test; the time spent (duration) sniffing by Cntnap2 knockout mice compared to controls in the home cage social target interaction test (C); the home cage two-choice sociability test (D); social preference test (E G); the time spent (duration) in each compartment (E) and approach duration (F) of Cntnap2 knockout mice compared to control in the three-chamber social test. The time spent (duration) for sniffing by Cntnap2 knockout mice compared to controls in the home cage two-choice social preference test (G). The number of animals per condition is as follows: Cntnap2 KO and WT in three-chamber social test, $\mathrm{n}=12$; Cntnap2 KO and WT in the home cage social target interaction test, $n=8$ and $n=9$, respectively; Cntnap $2 \mathrm{KO}$ and WT in the home cage two-choice sociability and social preference test, $\mathrm{n}=11$ and $\mathrm{n}=12$. respectively. All data are expressed as the mean \pm SEM. ${ }^{*} \mathrm{p}<0.05,{ }^{* *} \mathrm{p}<0.01,{ }^{* *} \mathrm{p}<0.001$, WT, wild-type group mice; Cntnap2 KO, Cntnap2 knockout mice. 
area (Fig. 4F, $\mathrm{p}=0.0416$ ) compared to controls. The compartment itself, and the approach duration to the familiar mouse area were not significantly different from controls (Fig. $4 \mathrm{E}$ and $4 \mathrm{~F}, \mathrm{p}>0.05$ ). In the home cage two-choice social preference test, NMDA-injected mice spent less time sniffing the novel stimulus mouse (Fig. 4G, $\mathrm{p}=0.0019$ ). However, there was not any significant difference in the sniffing duration of the familiar mouse cage between NMDAtreated and control groups (Fig. 4E, 4F and 4G, p>0.05). The results show that both three-chamber and home cage social tests can differentiate the social impairment of the NMDA-injected mice from the vehicle-treated mice.

\section{MK-801-injected mice exhibit reduced social interaction in both three-chamber and home cage social interaction tests}

MK-801-injected models have previously been used as a pharmacologic or drug-induced model of social deficits [32]. In the three-chamber social test, MK-801-injected mice spent significantly less time in the stranger compartment compared with their control counterparts (Fig. 5A, p=0.0114), whereas their time spent in the center chamber was greater (Fig. 5A, p=0.0492). In addition, the approach duration to the stranger mouse was reduced in the MK-801-injected mice (Fig. 5B, p=0.0021), while the approach duration to the empty compartment was not statistically different (Fig. 5A, 5B and 5C, $\mathrm{p}>0.05$ ).

In the home cage social target interaction test, MK-801-injected mice exhibited significantly reduced sniffing duration to the stimulus mouse during the entire 15 -min trial period (Fig. 5C, $\mathrm{p}=0.0194)$. Similarly, in the home cage two-choice sociability test, the MK-801-injected mice demonstrated significantly reduced sniffing duration of the stranger mouse (Fig. 5D, $\mathrm{p}=0.0002$ ), with more time spent sniffing the inanimate object versus controls (Fig. $5 \mathrm{D}, \mathrm{p}=0.0156$ ).

In the social novelty preference test using the three-chamber setup, the time spent in the novel mouse area and the approach area were significantly reduced in MK-801-injected mice (Fig. 5E, $\mathrm{p}=0.0065$; Fig. $5 \mathrm{~F}, \mathrm{p}=0.0003$, respectively). In contrast, there were no significant differences in the time spent in the center and familiar compartments, as well as its approach area (Fig. 5E, $\mathrm{p}>0.05$; Fig. 5F, $\mathrm{p}>0.05$, respectively). In the home cage two-choice social preference test, MK-801-injected mice spent less time sniffing the novel stimulus mouse than control mice (Fig. 5G, $\mathrm{p}=0.0029$ ), whereas there was no significant difference in the sniffing duration of the familiar mouse cage (Fig. 5G, p >0.05). The results show that both three-chamber and home cage social tests can differentiate the social impairment of the MK-801-injected mice from the vehicle-treated mice.
Cntnap2 gene knockout mice exhibit reduced social interaction in both the three-chamber and home cage social interaction test

Cntnap2 knockout (KO) mice were used as a genetic model of social deficit [33]. In the three-chamber social test, Cntnap2 KO mice spent less time in the stranger compartment than wild-type mice (Fig. 6A, $\mathrm{p}=0.0008$ ). Moreover, the time spent in the center and empty compartments was greater than controls (Fig. 6A, $\mathrm{p}=0.0003$ in the center compartment and $\mathrm{p}=0.0356$ in the empty compartment). In addition, the approach duration to the stranger mouse was reduced in Cntnap2 KO mice (Fig. 6B, p <0.0001), while the approach duration to the empty wire cage was not significantly different (Fig. 6B, p>0.05). Both the home cage social target interaction test and the home cage two-choice sociability test revealed significantly reduced sniffing duration by Cntnap $2 \mathrm{KO}$ mice of the stranger mouse (Fig. 6C and 6D, p=0.0002 in the home cage social target interaction test and 0.0023 in the home cage two-choice sociability test). Also, the Cntnap2 $\mathrm{KO}$ mice displayed reduced sniffing duration of the wire cage of the novel object in the home cage two-choice sociability test (Fig. 6D, p=0.0201).

In the social novelty preference test using the three-chamber set up, the Cntnap2 KO mice spent less time in the novel mouse compartment and more time in the center compartment than the wildtype mice (Fig. 6E, p=0.0218 in novel mouse compartment and $\mathrm{p}=0.0002$ in the center compartment). Furthermore, the Cntnap2 $\mathrm{KO}$ mice spent less time in the approach area of the novel mouse (Fig. 6F, $\mathrm{p}=0.0047$ ). The home cage two-choice social preference test also revealed reduced sniffing duration of the novel mouse by the Cntnap2 KO mice (Fig. 6G, p=0.0263). Nevertheless, there was no significant difference in sniffing of the familiar mouse between the Cntnap2 $\mathrm{KO}$ and wild-type mice (Fig. 6G, p>0.05). The results show that both the three-chamber and home cage social tests can differentiate the social impairment of a mutant mouse model (Cntnap2 KO) from wild-type mice.

\section{DISCUSSION}

In this study, we established a modified method to quantify the sociability and preference for social novelty of mice in a home cage setting using ethological measurement i.e., sniffing of a stimulus mouse inside a wire cage. This method follows the principle of initiating the social interaction or approach by the subject, and not the stimulus mouse, by simply measuring their social interaction ability [25]. In addition, the classic method can be benefitted or improved by a more sensitive measure of social behavior in a less stressful and more naturalistic environment [28, 29, 34, 35]. Thus, the current proposition arose from the hypothesis that factors 
of environmental novelty could induce some masking of social behaviors by novelty-seeking or increased exploratory behaviors. In addition, the classic three-chamber assay exposes the grouphoused mice into isolation-induced behaviors during the test [26, 36]. The differential time points of the experiment for each subject and for each group, which makes the complete experimental period time-consuming, is also an element that needs to be considered.

The current social interaction methods used in the home cage setting could address these issues. First, the group-housed mice can be tested together in their home environment in a time-efficient manner. During the direct observation of social behaviors, all cage mates can serve as subject mice at the same time - they each have equal opportunity to interact with the stimulus mice. The round design and placement of the wire cage in the center of the cage (in the home cage social target interaction test) during the test makes it possible for each mouse to sniff the cage at the same time. In addition, we designed the opening to the caged stimulus mouse in the home cage two-choice sociability and social preference test to have sufficient space for at least five mice to sniff at the same time. This allows high throughput screening of behaviors simultaneously for various subjects. Secondly, the element of having a familiar environment allows subject mice to focus more on the novel stimulus, and less on exploring other areas. According to our results, as well as in previous studies, testing the subject mice's social interaction in a novel environment, that is, a new cage, increases their locomotor or exploratory activities [37] and reduces their sniffing duration of the stranger mice compared to home cage tested mice. In other cases, some rodents also develop anxiety-related behaviors such as freezing, grooming, and thigmotaxis [38]. These behaviors could directly affect the interest of new cage tested mice in interacting with a stranger mouse. The other novel factors in this test, aside from the stimulus mouse, are the wire cage where the stimulus mouse is enclosed, and the mounting of home cages in the testing area. Both of these factors benefitted from a 30 min habituation period, where it was shown that the interest of subject mice in the introduced wire cage decreases over time (familiarization). This then allows the initiation of novelty-induced interest in the stranger mouse thereafter. The reason for using a stranger stimulus mouse over a familiar mouse is the reduced active social interactions observed between cage mates [27]. Thus, in order to avoid the novelty-induced factor, a home cage setting is of good use for the study of social interaction in mice. Also, individual behaviors of mice can be affected by the circadian rhythm during the time of testing, and so the testing of mice in groups addresses this time difference issue.

The home cage social interaction tests assess the sociability or social preference of subject mice to the stimulus mouse through the measurement of sniffing duration of the wire cage. Technically, the type or aspect of social behavior we are measuring in this new configuration is identical to the three-chamber social test, except that we used the entire set of cage mates simultaneously and in their home cage while the test was being performed. The threechamber social test mainly compares the sociability or social preference of subject mice by measuring the time spent by the subject mouse in each chamber during the $10 \mathrm{~min}$ trial period. In some cases, the sniffing of the wire cage by a manual observer or the time spent in a specified interaction/approach zone in close proximity to the wire cage were also presented $[26,39]$. In the home cage social interaction test, we measured social interaction based only on the sniffing duration of the wire cage. Although there may be a slight difference in the parameters, depending on what other researchers use for the three-chamber social assay, both this and the home cage social interaction tests assess the sociability or the social preference of mice, given that the direct measurement of sniffing duration is more specific and ethological.

It should be noted that during the test, we did not observe any social aggression of the subject mice toward each other, as most of them were preoccupied interacting with the stimulus mouse. We also used adolescent mice (from 3 7 weeks) for this test, which is relevant to social deficit disorders such as autism. At these ages, aggressive behaviors were less frequently observed. Another concern of group testing the mice in the home cage social interaction test is that each mouse might affect or dilute the behaviors of other mice during the experiment. However, it was previously reported that group-housed mice with an identified social hierarchy also differed in behavior performance, even in some individualized tests [40]. Thus, whether we use individual or group testing, the innate propensity of each mouse based on the social hierarchy and many other factors will still influence the behavior performance in any test.

Based on our results, while the three-chamber assay reveals the reduction of social behaviors in the three ASD models used (based on their time spent in chamber and approach duration parameters), the home cage social interaction test can similarly demonstrate and complement those differences. To rule out whether the social behavior of mice in group-testing may affect the individual behavior of those mice, we performed preliminary experiments by mixing wild-type and $\mathrm{KO}$ mice. Based on the available number of animals, we housed one Cntnap2 KO mouse together with three wild-type mice, and three Cntnap2 KO mice together with one wild-type mouse to compare their social performance in the home cage social interaction test. The results indicate that both housing conditions can still show a differential response between the mutant and wild-type mice, where the Cntnap2 KO mice have 
reduced social sniffing to the wire cage compared to the vehicletreated mice (data not shown).

The use of the three-chamber assay, or any other type of social test, as the lone basis for the conclusion that a certain mouse model is socially impaired or not, might give room for errors and false negative results. Indeed, a few studies that have introduced or established a new model of autistic behaviors have shown no difference in the three-chamber assay parameters, but had an obvious difference in other social related tests, such as juvenile social play or reciprocal social interactions [41]. Therefore, it is recommended that a series of behavior assays, especially including more naturalistic home cage settings to assess the social behavior repertoire of mice models with social deficiency, would be optimal to draw conclusions. It should be noted that our new methodology did not try to compare to the three-chamber analysis as more effective in terms of social impairment outcomes. Our purpose is to present a new method of social behavior test that is quick and easy to perform for high throughput screening purposes.

The home cage social interaction test is useful in screening the validity of new models of ASD. In addition, it can serve as a complementary and supplementary method to already established social paradigms, with the advantage of a naturalistic environment and the ability to test the subjects simultaneously in groups. Furthermore, this task can be an effective tool as a high throughput screening for new drug candidates to treat social deficits, and to screen out pharmacological models of social deficits relevant to neurodevelopmental disorders like ASD. Interestingly, this study also showed that both increased excitatory signaling (i.e., the NMDA induced model) or decreased excitatory signaling (i.e., the Cntnap2 KO and MK-801-injected models) cause social deficits in the home cage social interaction paradigm, which suggests that this method can be a versatile behavioral analysis tool to screen out ASD animal models with different etiological backgrounds. Therefore, we suggest the addition of home cage social test paradigms to screen out models of social behavior deficiency more efficiently and meaningfully.

\section{ACKNOWLEDGEMENTS}

This work was supported by the Korea Institute of Science and Technology (Grant No. 2E28412-18-119); the Bio \& Medical Technology Development Program of the National Research Foundation (NRF) funded by the Korean government (MSIT) (NRF-2017M3A9G2077568); and by the Business for Startup growth and technological development (TIPS Program grants No. S2525668) funded by Korea Ministry of SMEs and Startups in 2017.

\section{REFERENCES}

1. Robinson GE, Fernald RD, Clayton DF (2008) Genes and social behavior. Science 322:896-900.

2. House JS, Landis KR, Umberson D (1988) Social relationships and health. Science 241:540-545.

3. Uchino BN, Cacioppo JT, Kiecolt-Glaser JK (1996) The relationship between social support and physiological processes: a review with emphasis on underlying mechanisms and implications for health. Psychol Bull 119:488-531.

4. Geschwind DH (2008) Autism: many genes, common pathways? Cell 135:391-395.

5. Atladóttir HO, Pedersen MG, Thorsen P, Mortensen PB, Deleuran B, Eaton WW, Parner ET (2009) Association of family history of autoimmune diseases and autism spectrum disorders. Pediatrics 124:687-694.

6. Shibayama A, Cook EH Jr, Feng J, Glanzmann C, Yan J, Craddock N, Jones IR, Goldman D, Heston LL, Sommer SS (2004) MECP2 structural and 3囚-UTR variants in schizophrenia, autism and other psychiatric diseases: a possible association with autism. Am J Med Genet B Neuropsychiatr Genet 128B:50-53.

7. Valenti D, de Bari L, De Filippis B, Henrion-Caude A, Vacca RA (2014) Mitochondrial dysfunction as a central actor in intellectual disability-related diseases: an overview of Down syndrome, autism, Fragile X and Rett syndrome. Neurosci Biobehav Rev 46:202-217.

8. Derecki NC, Privman E, Kipnis J (2010) Rett syndrome and other autism spectrum disorders--brain diseases of immune malfunction? Mol Psychiatry 15:355-363.

9. Mouridsen SE, Rich B, Isager T (2010) A longitudinal study of gastrointestinal diseases in individuals diagnosed with infantile autism as children. Child Care Health Dev 36:437-443.

10. Kaufmann WE, Cortell R, Kau AS, Bukelis I, Tierney E, Gray RM, Cox C, Capone GT, Stanard P (2004) Autism spectrum disorder in fragile $\mathrm{X}$ syndrome: communication, social interaction, and specific behaviors. Am J Med Genet A 129A:225234.

11. Lord C, Bishop SL (2009) The autism spectrum: definitions, assessment and diagnoses. Br J Hosp Med (Lond) 70:132-135.

12. Luyster R, Gotham K, Guthrie W, Coffing M, Petrak R, Pierce K, Bishop S, Esler A, Hus V, Oti R, Richler J, Risi S, Lord C (2009) The Autism Diagnostic Observation Schedule-toddler module: a new module of a standardized diagnostic measure for autism spectrum disorders. J Autism Dev Disord 39:13051320 .

13. Mundy P, Sigman M, Ungerer J, Sherman T (1986) Defining 
the social deficits of autism: the contribution of non-verbal communication measures. J Child Psychol Psychiatry 27:657669.

14. Lord C, Risi S, Lambrecht L, Cook EH Jr, Leventhal BL, DiLavore PC, Pickles A, Rutter M (2000) The autism diagnostic observation schedule-generic: a standard measure of social and communication deficits associated with the spectrum of autism. J Autism Dev Disord 30:205-223.

15. Krishnan V, Han MH, Graham DL, Berton O, Renthal W, Russo SJ, Laplant Q, Graham A, Lutter M, Lagace DC, Ghose S, Reister R, Tannous P, Green TA, Neve RL, Chakravarty S, Kumar A, Eisch AJ, Self DW, Lee FS, Tamminga CA, Cooper DC, Gershenfeld HK, Nestler EJ (2007) Molecular adaptations underlying susceptibility and resistance to social defeat in brain reward regions. Cell 131:391-404.

16. Sano H, Nagai Y, Miyakawa T, Shigemoto R, Yokoi M (2008) Increased social interaction in mice deficient of the striatal medium spiny neuron-specific phosphodiesterase 10A2. J Neurochem 105:546-556.

17. Rodriguiz RM, Chu R, Caron MG, Wetsel WC (2004) Aberrant responses in social interaction of dopamine transporter knockout mice. Behav Brain Res 148:185-198.

18. Zhan Y, Paolicelli RC, Sforazzini F, Weinhard L, Bolasco G, Pagani F, Vyssotski AL, Bifone A, Gozzi A, Ragozzino D, Gross CT (2014) Deficient neuron-microglia signaling results in impaired functional brain connectivity and social behavior. Nat Neurosci 17:400-406.

19. Avale ME, Chabout J, Pons S, Serreau P, De Chaumont F, Olivo-Marin JC, Bourgeois JP, Maskos U, Changeux JP, Granon S (2011) Prefrontal nicotinic receptors control novel social interaction between mice. FASEB J 25:2145-2155.

20. Silverman JL, Yang M, Lord C, Crawley JN (2010) Behavioural phenotyping assays for mouse models of autism. Nat Rev Neurosci 11:490-502.

21. Bolivar VJ, Walters SR, Phoenix JL (2007) Assessing autismlike behavior in mice: variations in social interactions among inbred strains. Behav Brain Res 176:21-26.

22. Terranova ML, Laviola G, Alleva E (1993) Ontogeny of amicable social behavior in the mouse: gender differences and ongoing isolation outcomes. Dev Psychobiol 26:467-481.

23. Wesson DW (2013) Sniffing behavior communicates social hierarchy. Curr Biol 23:575-580.

24. Nadler JJ, Moy SS, Dold G, Trang D, Simmons N, Perez A, Young NB, Barbaro RP, Piven J, Magnuson TR, Crawley JN (2004) Automated apparatus for quantitation of social approach behaviors in mice. Genes Brain Behav 3:303-314.

25. Moy SS, Nadler JJ, Perez A, Barbaro RP, Johns JM, Magnuson
TR, Piven J, Crawley JN (2004) Sociability and preference for social novelty in five inbred strains: an approach to assess autistic-like behavior in mice. Genes Brain Behav 3:287-302.

26. Yang M, Silverman JL, Crawley JN (2011) Automated threechambered social approach task for mice. Curr Protoc Neurosci Chapter 8:Unit 8.26.

27. Fairless AH, Katz JM, Vijayvargiya N, Dow HC, Kreibich AS, Berrettini WH, Abel T, Brodkin ES (2013) Development of home cage social behaviors in BALB/cJ vs. C57BL/6J mice. Behav Brain Res 237:338-347.

28. de Visser L, van den Bos R, Kuurman WW, Kas MJ, Spruijt BM (2006) Novel approach to the behavioural characterization of inbred mice: automated home cage observations. Genes Brain Behav 5:458-466.

29. Kas MJ, Van Ree JM (2004) Dissecting complex behaviours in the post-genomic era. Trends Neurosci 27:366-369.

30. Lee JH, Zhang JY, Wei ZZ, Yu SP (2018) Impaired social behaviors and minimized oxytocin signaling of the adult mice deficient in the N-methyl-d-aspartate receptor GluN3A subunit. Exp Neurol 305:1-12.

31. Hong W, Kim DW, Anderson DJ (2014) Antagonistic control of social versus repetitive self-grooming behaviors by separable amygdala neuronal subsets. Cell 158:1348-1361.

32. Rung JP, Carlsson A, Rydén Markinhuhta K, Carlsson ML (2005) (+)-MK-801 induced social withdrawal in rats; a model for negative symptoms of schizophrenia. Prog Neuropsychopharmacol Biol Psychiatry 29:827-832.

33. Peñagarikano O, Abrahams BS, Herman EI, Winden KD, Gdalyahu A, Dong H, Sonnenblick LI, Gruver R, Almajano J, Bragin A, Golshani P, Trachtenberg JT, Peles E, Geschwind DH (2011) Absence of CNTNAP2 leads to epilepsy, neuronal migration abnormalities, and core autism-related deficits. Cell 147:235-246.

34. Casadesus G, Shukitt-Hale B, Joseph JA (2001) Automated measurement of age-related changes in the locomotor response to environmental novelty and home-cage activity. Mech Ageing Dev 122:1887-1897.

35. Koot S, Adriani W, Saso L, van den Bos R, Laviola G (2009) Home cage testing of delay discounting in rats. Behav Res Methods 41:1169-1176.

36. Kaidanovich-Beilin O, Lipina T, Vukobradovic I, Roder J, Woodgett JR (2011) Assessment of social interaction behaviors. J Vis Exp 2473.

37. Eilam D, Golani I (1989) Home base behavior of rats (Rattus norvegicus) exploring a novel environment. Behav Brain Res 34:199-211.

38. Westbrook RF, Good AJ, Kiernan MJ (1994) Effects of the 
interval between exposure to a novel environment and the occurrence of shock on the freezing responses of rats. Q J Exp Psychol B 47:427-446.

39. Kim JW, Park K, Kang RJ, Gonzales EL, Kim DG, Oh HA, Seung H, Ko MJ, Kwon KJ, Kim KC (2019) Pharmacological modulation of AMPA receptor rescues social impairments in animal models of autism. Neuropsychopharmacology 44:314-323.

40. Horii Y, Nagasawa T, Sakakibara H, Takahashi A, Tanave A,
Matsumoto Y, Nagayama H, Yoshimi K, Yasuda MT, Shimoi K, Koide T (2017) Hierarchy in the home cage affects behaviour and gene expression in group-housed C57BL/6 male mice. Sci Rep 7:6991.

41. Hamilton SM, Green JR, Veeraragavan S, Yuva L, McCoy A, Wu Y, Warren J, Little L, Ji D, Cui X, Weinstein E, Paylor R (2014) Fmr1 and Nlgn3 knockout rats: novel tools for investigating autism spectrum disorders. Behav Neurosci 128:103109. 